\title{
Revisited Leonardo Fibonacci law of golden mean as surface-centric approach for form sustainable in design
}

\begin{abstract}
The Golden Proportion is also known as the Golden Mean, Phi, or Divine Proportion, this law was made famous by Leonardo Fibonacci around 1200 A.D. He noticed that there was an absolute ratio that appears often throughout nature, a sort of design that is universally efficient in living things and pleasing to the human eye. The most renowned and influential product designer to work with such grid systems is Dieter Rams. Rams established a philosophy that was applied by Braun products that focused on providing clarity of function by avoiding superfluous. The divine proportion also has been used by companies like Apple to design products by Jonathan Ive, it is said to have been used by Twitter to create their new profile page, and has been used by major companies all over the world to design logos. The aim of this study is to assess whether the surface-centric approach has emotional effect to users and the objectives are to examine the relationship between the surface-centric approach and good design in the relationship between the surface-centric approach and consumer behaviour. This study is important as the framework will contribute to the body of knowledge in design methodology in search for ideas in form giving during designing process. It will also revive the approach of golden section in product design area.
\end{abstract}

Keyword: Golden proportion; Surface-centric approach; Divine proportion 